\title{
Applications of Planar Transmission Lines
}

\author{
Parnika Gupta, Khushi Arora, Shefali Chopra \\ Department of Electronics and Communication Engineering \\ Manav Rachna University
}

\begin{abstract}
The transmission lines are basically used in two ways either as a medium for signal transmission or as a circuit component. They can also be designed as impedance matching sources for antennas or stubs filters. The ease of fabrication of devices that use transmission lines for interconnection among the other sub-components of the circuit gives it an edge over the other tedious wiring methods. Planar transmission lines specifically stand out in this regard with respect to the latest fabrication technology being used for the nanoscale development of integrated circuits and at the same time playing a major role in radio frequency communication systems.
\end{abstract}

Index Terms-Antenna arrays, Band pass filter, Coupler, Power splitter, Resonators.

\section{INTRODUCTION}

The planar transmission lines and especially the microstrip lines has been a major field of study over the recent years. It has also attracted a lot of attention to its advantage of being modified to a patch antenna. The development of planar transmission lines for broadband communication suitability as well as for the manufacturing of monolithic microwave integrated circuits gives us a clear picture of the range of its applications and benefits.

\section{APPLICATIONS OF MICROSTRIP LINES}

A microstrip line is referred to as the quasi-TEM transmission line in which the microstrip is embedded on the surface of an insulating dielectric substrate with a ground plane on its parallel side as shown in Fig.1. These microstrip lines have an easily accessible top surface so that further fabrication of devices is highly convenient. But the losses due to the radiations and electromagnetic interference as well as the dimensions of the field external to the dielectric material makes it a source of technology to be used within constrained design. The dielectric material being used comprises of GaAs, Silicon etc. and it displays a Q-factor of 250 when the radiations are low and the Q-factor lies in the range of 100 to 150 for high radiations. Being small sized and easily integrable, these lines are used for a wide range of microwave circuit designs (MICs) like printed circuit lines for miniaturized microwave front end applications on the receiver side which includes devices such as mixers .These mixers are composed of microwave ring circuits containing balanced microstrip lines. In millimeter wave applications low power stages of integrated transmitter system [1] as well as enhancement gain effects of power amplifiers adhering to CMOS transceivers for use in wireless communication [2] are also acknowledged to microstrip line applications in microwave circuits .The Wideband L-Band Suboptimum Class-E power amplifiers for RF and satellite communication are also structured with microstrip lines[3] .The microstrip technology is also tapped for Low pass L-Band microwave

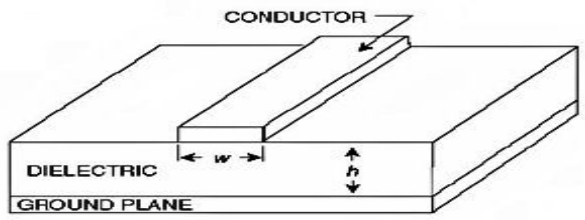

Figure 1: Microstrip Line

filters (stepped impedance microstrip lines)and modifying the characteristic impedance, conductor shape and dielectric material to improve circuit performance in communication systems[4].The microstrip band pass filter is employed in S-band of radio frequency for WiMAX (Worldwide Interoperability for Microwave Access) Application [5] .The shielded microstrip lines are the microstrip lines that are shielded by a metallic enclosure so that the electromagnetic interference losses are reduced and these are then casted for designing of microwave filters . These lines are also applied as couplers or adaptors for measurement of impedance or VSWR of electromagnetic devices[6].

\section{APPLICATIONS OF STRIPLINES}

Stripline (or suspended stripline) is a transmission line in which the conducting portion is immersed in a dielectric material with the metallic ground planes on either side of the dielectric which are mostly shorted to avoid the fields from escaping the dielectric substrate which emits radiation on a low scale, boasts on supporting a Q factor of about 500 and its load impedance may vary from 40 to 150 ohms.Fig. 2 shows the above mentioned details of the stripline. The stripline accommodates the need for providing isolation among the various circuit sub-parts in a multi-layered circuit board though chip mounting is relatively difficult. The added advantage to a stripline is that its impedance can be varied to a larger extent as compared to that of microstrip lines because they allow more flexibility in the matter of positioning the conductor within the dielectric substrate. For the image decoding applications in the human head, where data obtained from transceiver array loops was juxtaposed with the data obtained from stripline transmission line array for 7 Tesla Parallel Imaging striplines were used [7].The Band pass filters are designed by utilizing the parallel coupled stripline stepped impedance resonator which controls the responses 
and losses encountered by the resonators [8] since it can be used over a broad frequency range. Double conductor stripline and shielded stripline is also developed for use in the less tapered configurations while manufacturing of integrated circuits and where the circuit is susceptible to noise or signal distortion. Earlier, the striplines were considered a subpart of the microstrip line but over the years it has succeeded in carving out its own place in fabrication technology due to the presence of the conductor trace inside the substrate.

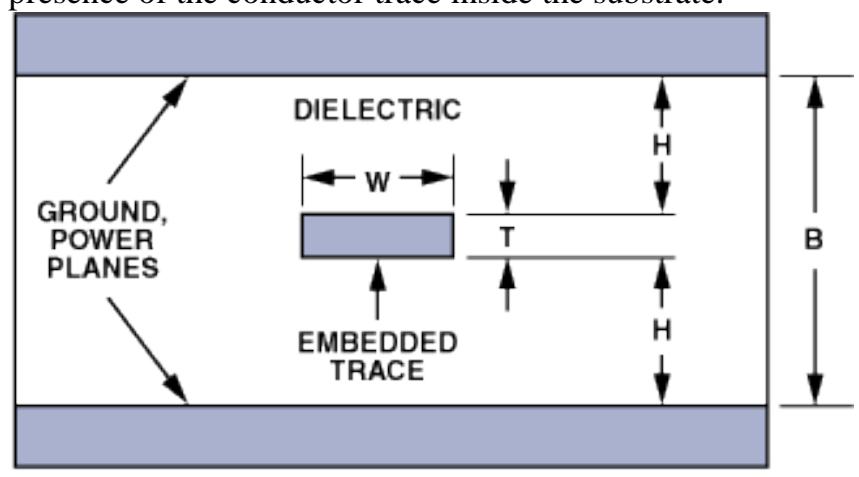

Figure 2: Stripline

\section{APPLICATIONS OF SLOTLINES}

The slotline has a substrate whose one side remains as it is and the other side has a ground plane on which a narrow slot is

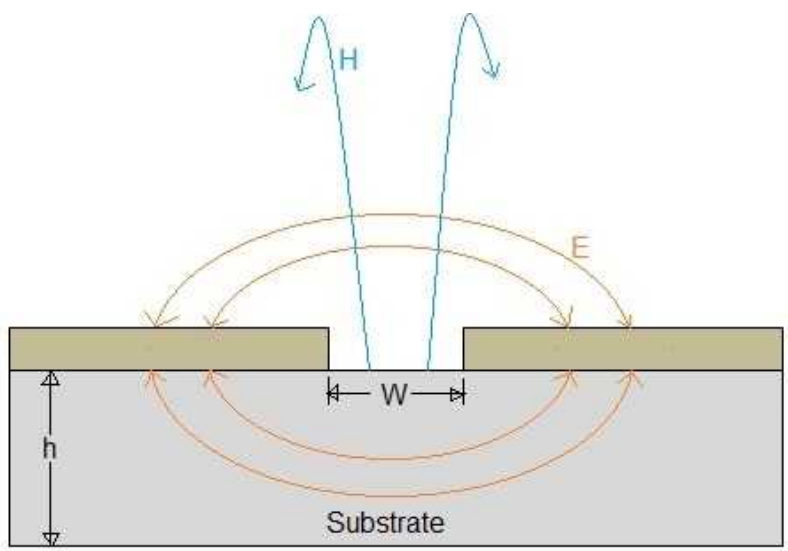

Figure 3: Field Configuration in a slotline

etched. It doesn't support TEM mode and is used in conjunction with microstrip line for designing circuits and it radiates moderately, has a high dispersion rate, supports a Q factor of about 100 which is less than that of the stripline and its impedance varies over a range of 60 to $200 \mathrm{ohms}$. The field density is limited within the substrate so, the higher order modes are suppressed as shown in Fig. 3 and it supports the TE mode which is known for its diffusive nature so it is mostly not recommended for broadband applications except for Bilateral slotline or double slotline in which twin slot lines are etched on both sides of the substrate as it proffers less impedance and matching with stripline. When one end of the slotline is shorted its characteristics are similar to that of a half-wave resonator and by introducing a MEMS switch at the other end of the slot line transmission line, it can control the load of the slotline and thus microelectromechanical switches are used for application in the tunable resonator(digital frequency tuning).Annular rings generated from slotlines such as slot line ring resonators, slotline dual-mode Band Pass filters are taken as a part of the circuitry in resonator filter and coupler applications which is significantly useful for MICS and MMICS[9].The millimeter wave technology which is apt for Wireless Communication Networks uses integrated NRD(Non-Radiative Dielectric) Guide and slotline for transmission of frequencies in the millimeter wave frequency region[10]. Certain changes are also being made to the traditional TSA(Tapered Slot Antennas) which is a kind of printed antenna for gain enhancement to be used at different frequencies for outer space communication as well as domestic communication purposes[11].The hybrid T-Junctions are also devised through the transition of Microstrip lines to slotlines which are further extracted for other circuit components such as power dividers, mixers and modulators such as ASK modulator, phase detector( since there is minimum amount of signal loss in the case of isolated coupled slotline). Due to an acceptable level of non-interference between balanced and unbalanced slotlines it also finds uses in feeding applications in rat-race hybrid junction, pulse inverters, couplers etc. with broader bandwidth graphs.

\section{APPLICATIONS OF FINLINES}

\section{Rectangular Waveguide}

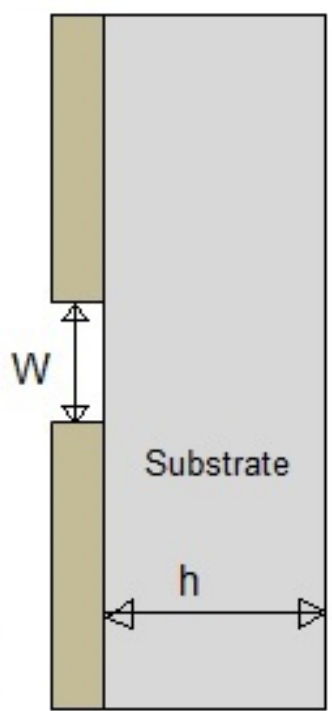

Figure 4: Finline

Finlines are basically the slotlines that are placed in the interior E-plane section of a rectangular waveguide which is evident from Fig 4. The substrate has low dielectric constant and it sustains a combination of TE and TM modes thus leading to low dispersion. It also yields circularly polarized fields that assist the working of non-reciprocal devices such as isolators and circulators. They emit the least amount of radiation or in some ideal cases -no radiations and their Q-factor is 500 . The characteristic impedance is between 20 ohms and 400 ohms. Integrated finlines are mostly operated in the frequency range of 30 to 100 Gigahertz because at high frequencies there are lesser number of design constraints and these structures are used for low and medium power applications. The finline PIN diode switches as well as finline PIN diode attenuators in millimeter wave technology like Ultra Broadband PIN diode switches, PIN diode attenuators working in Ka-Band, Q-Band and W-Band. BPSK and QPSK modulators are also constructed from finline PIN diode phase 
shifter and finline coupler and this technology further finds applications in communication systems in addition to balanced mixers using waveguide to Microstrip transition [12].

\section{APPLICATIONS OS COPLANAR WAVEGUIDES}

In coplanar waveguides (CPW), a conductor strip and two ground planes are placed on the same plane above the substrate as proposed by Wen. The design of a coplanar waveguide is shown in Fig.5. The grounded coplanar waveguide (GCPW) has a ground plane below the substrate. The field is mostly confined within the thick substrate leading

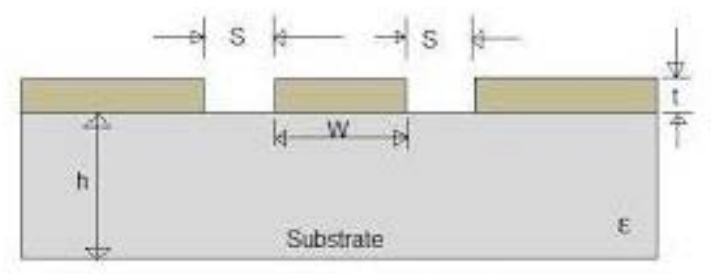

Figure 5: Coplanar Waveguide

to less dispersion and making CPWs the preferred choice for MMIC fabrication using GaN technology(Gallium Nitride based devices). CPW supports quasi TEM mode at low frequencies and TE mode at high frequencies leading to their application in microwave Integrated circuits (MICs). The impedance values range from 20 ohms to 250 ohms and the conductor strip can easily be tapered to a pin for facilitating attachable circuits in broadband communication. GCPWs are also applied in isolated RF switches, power splitters, filters and directional couplers due to the high degree of isolation between circuit components. Coplanar waveguide resonators are developed using temperature variations in superconducting material for detector applications [13]. MEMS switches (Microelectromechanical switches) applied in capacitive metal membrane shunt configuration and CPW resonator for $\mathrm{X}$ Band pass filters prefer $\mathrm{CPW}$ because of the presence of both the ground as well as conductor on the same plane [14-16].Waveguide to coplanar transitions are employed for communication in millimeter and centimeter frequency range [15]. Grounded CPW (GCPW) lines are used as pickup structures in Energy Beam Position Monitors operated within particle accelerators used Grounded CPW (GCPW) for the ease of transition to coaxial connectors later in the operation [17].

\section{CONCLUSION}

Further advancements are being made by scientists and engineers all over the globe to improve the quality of transmission lines by variation in its characteristic properties. Already we are using optical fiber transmission for high-speed communication and this transmission speed is being improved for wireless communication using planar transmission lines.

\section{REFERENCES}

[1] Prof. Usha Yadav, "Simulation and Design Analysis of Integrated Transmitter System for Millimeter Wave Applications," International Journal of Advanced Research in Computer Science and Software Engineering, Volume 5, Issue 3, March 2015.

[2] Sandeep, Neeraj, Mohit Sharma , "Enhancement Gain of CMOS PA using Microstrip line for Millimeter wave applications," INTERNATIONAL JOURNAL FOR RESEARCH IN APPLIED SCIENCE AND ENGINEERING TECHNOLO GY (I JRAS ET), Vol. 2 Issue VI, June 2014, ISSN: 2321-9653.

[3] Francisco Javier Ortega-Gonzalez, David Tena-Ramos, Moises Patiño-Gomez, Jose Manuel Pardo-Martin, and Diego Madueño-Pulido, "High-Power Wideband -Band Suboptimum

Class-E Power Amplifier," IEEE Trans. on Microwave Theory and Techniques, VOL. 61, NO. 10, OCTOBER 2013.

[4] Omid Borazjani and Arman Rezaee, "Design, Simulation and Construction of a Low Pass Microwave Filters on the Micro Strip Transmission Line," International Journal of Computer Theory and Engineering, Vol. 4, No. 5, October 2012.

[5] Avishek Das, Avisankar Roy, Kushal Roy, Amit Bhattacharyya, Dibyendu Chowdhury, "Design and Analysis of Microwave Planar Band Pass Filter for WiMAX Application," International Journal of Emerging Trends \& Technology in Computer Science (IJETTCS), Volume 3, Issue 6, November-December 2014, ISSN 2278-6856.

[6] Roddy, Frank M. ; Sandia Corporation Albuquerque, New Mexico; Hewitt, Jack G, ".Use of Microstrip Transmission Line to Improve Broadband Electromagnetic Measurements,"

Electromagnetic Compatibility Symposium Record, 1969 IEEE,

17-19 June 1969.

[7] Adriany G, Van de Moortele PF, Wiesinger F, Moeller S, Strupp $J P$, Andersen $\quad P$, Snyder $\quad C$, Zhang $X$, Chen $W$, Pruessmann $K P$, Boesiger $P$, Vaughan $T$, Uğurbil $K$, "Transmit and Receive Transmission Line Arrays for 7 Tesla Parallel Imaging," Magnetic Resonance in Medicine 53:434-445 (2005).

[8] Makimoto, Mitsuo ; Sadahiko Yamashita, "Bandpass Filters Using Parallel Coupled Stripline Stepped Impedance Resonators,"

Microwave Theory and Techniques, IEEE Transactions on (Volume: 28, Issue: 12 ).

[9] Chien-Hsun Ho, Lu Fan, Kai Chang, "Slotline Annular Ring Elements and Their Applications to Resonator, Filter and Coupler Designs," IEEE Trans. On Microwave Theory and Techniques, VOL. 41, NO. 9, SEPTEMBER 1993.

[10] Nagendra P. Pathak, "Integration of NRD Guide and Slot Line for Millimeter Wave Indoor Wireless Applications," Proceedings of WFMN07, Chemnitz, Germany.

[11] Wen-Xun Zhang, "Modified Tapered Slot-line Antennas for Special Applications," REV Journal on Electronics and Communications, Vol. 2, No. 3-4, July - December, 2012.

[12] He Liquan, Wu Xidong, Zhu Xiaowei, "Fin-line Pin diode BPSK and QPSK modulators," Waves, February 1993, Volume 14, Issue 2, pp 355-361.

[13] G Hammer, S Wuensch, M Roesch, K Ilin, E Crocoll and M Siegel, "Superconducting coplanar waveguide resonators for detector applications," Superconductor Science and Technology Volume 20 Number 11,G Hammer et al 2007 Supercond.Sci.Technol. 20 S408 doi:10.1088/0953-2048/2 $0 / 11 / \mathrm{S} 21$.

[14] Rainee N. Simons, NASA Glenn Research Center, Cleveland, Ohio, "Coplanar Waveguide Circuits, Components and Systems".

[15] G.C. Dalman, "New waveguide-to-coplanar waveguide transition for centimeter and millimeter wave applications," Electronics Letters, Volume 26, Issue 13, 21 June 1990, p. $830-$ 831,DOI: $10.1049 / \mathrm{el}: 19900544$, Print ISSN 0013-5194, Online ISSN 1350-911X. 
[16] Mihai şOVA, Ion BOGDAN, "Coplanar Waveguide Resonator Design for Array Antenna Applications," Mikrotalasna revija, December 2003.

[17] A. Penirschket, A. Angelovski, R. Jakoby, Institut $f$ "ur Mikrowellentechnik und Photonik, TU Darmstadt, Germany, Sydlo, U. Mavric, C. Gerth, S. Vilcins-Czvitkovits, D. Noelle DESY, Hamburg, Germany, "GROUNDED COPLANARWAVEGUIDE TRANSMISSION LINES ASPICKUPS FOR BEAM POSITION MONITORING IN PARTICLE ACCELERATORS,"TUPC29, Proceedings of IBIC2013, Oxford, UK.

[18] Image citations: www.embedded.com, www. analog.com, www. RF Wireless World.com. 